\title{
Editorial
}

\section{Electronic journals: looking ahead}

Scientific journals have migrated onto the world wide web at an astonishing rate. They've had no option: scientific researchers have moved even faster. For researchers, information that is out of sight is out of mind. Not to be seen is not to be cited and not to be cited is not to be read. It's a terminally vicious circle for journals that are not online. And yet, once readers have recovered from the minor shock of seeing an article on a screen instead of a page it's hard to tell the difference between the versions. The medium may have changed, but the basic unit of scientific communication looks the same. Revealingly, we still call it a paper.

Historians of modernisation tell us that this is what always happens. Take the arrival of automation, for example. The first phase is the electrification of processes: people do exactly what they did before, except the power source has changed. Next comes the phase of enhancement: unique features are added that would have been impossible before (in the case of the web, think search engine). Next comes the evolutionary phase: where what has been done routinely is transformed into something entirely different. This is when life on the web will get really exciting.

We're not there yet, but we do have an inkling of what it may be like. It's clear that we'll move from issue based publishing to article based publishing. There's no longer any reason to print a series of papers, bind them up together, and send them out between journal covers. Many electronic journals have begun to publish ahead of print, or even instead of print. Articles are published immediately after acceptance and technical editing. This will have profound affects on the economics of publishing, not to mention impact factors and citations (notions of volumes, issues, and page numbers are meaningless on the web).

The web is putting peer review, scientific journals' most sacred function of all, under increasing scrutiny. For over a decade now, high energy physicists have posted their completed research on an eprint server, before submitting it for peer review. Their community has shown a strong appetite for accessing this research, which is available fast, free, and in full text. The eprint server co-exists with traditional physics journals, in which the same research appears slowly, expensively, and after peer review. This shift has enlivened debates about the value of peer review far beyond the confines of the high energy physics community. Peer review itself could change. Already journals encourage postpublication peer review from readers in the form of letters to the editor. This same interest and energy could be harnessed to provide prepublication peer review of manuscripts, posted onjournal websites at submission.

Even if we accept the centrality of peer review as it's currently practised, the web allows radical restructuring of the process. Peer review and distribution - inextricably linked in paper journals - can now be uncoupled: one agency can perform the peer review; another can handle the distribution. BioMedCentral ${ }^{1}$ and the Public Library of Science ${ }^{2}$ are both moving towards agency models, whereby authors pay up front for peer review and distribution of their work, which will be made available free to endusers in perpetuity.

The web allows journals to have a much more intimate relationship with its readers. At the BMJ we routinely ask readers for their opinions on a whole range of issues, including the choice of theme issue topics for the year. ${ }^{3}$ Online polls are quicker and easier to conduct than their paper analogue; their results allow us to be more responsive and hopefully more useful to our readership.

If this reads like the prelude to the abandonment of paper for the web, it isn't. Nothing our readers have told us suggests that they want this. In a recent poll of BMA members (who receive the paper BMJ as a benefit of membership) 90 per cent strongly agreed or agreed with the statement that 'Despite the availability of the electronic journal, I want to keep receiving the paper journal.' While paper has such advantages over the computer screen (readability, portability, durability, cost) it would be irrational to propose otherwise. But there are useful things that websites can do which paper journals can't. We are beginning to explore this Aladdin's cave of possibilities and to work out how we can exploit the unique properties of both media. The future is likely to be 'and' rather than 'either/or'.

Tony Delamothe Web Editor bmj.com

\section{References}

1 http://www.biomedcentral.com

$2 \mathrm{http}: / /$ www.publiclibraryofscience.org

$3 \mathrm{http} / / / \mathrm{bmj} . \mathrm{com} / \mathrm{misc} / \mathrm{strawpolls.shtml}$ 